\title{
Seismicity of Nepal and the surrounding region
}

\author{
Dilli Ram Thapa
}

Department of Geology, Birendra Multiple Campus, Tribhuvan University, Nepal

\begin{abstract}
This study updates an earthquakes catalogue (in terms of surface-wave magnitude, $\mathrm{Ms} \geq 4.0)$ for Nepal and the surrounding region $\left(26^{\circ}-31.7^{\circ} \mathrm{N}\right.$, $79^{\circ}-90^{\circ} \mathrm{E}$ ) covering the period from 1255 to 2015 and analyze the spatio-temporal distribution patterns of seismicity. The spatial distribution of all catalogued earthquakes indicates that earthquakes are unevenly distributed in Nepal and the surrounding region with distinctly higher earthquake activity in the far western and eastern parts of Nepal than the southern part of the country. The temporal distribution of updated seismicity data (1255-2015) indicates that there are very little accounts of significant earthquakes prior to 1800. Furthermore, the temporal trend of the updated seismic catalogue clearly demonstrate the gradual increase in earthquake data during the time window 1801-1963 and considerable increase in number and quality of seismic events predominantly from 1964 onwards.
\end{abstract}

Keywords: Seismicity, Earthquake catalogue, Nepal

Accepted: 19 June 2018

\section{INTRODUCTION}

Nepal is one of the long-identified high seismic hazard nations lies in the central most segment of the Himalayan collided zone with a entire population exceeded 26 million inhabitants (CBS 2011). During its history, Nepal has experienced six large/great damaging earthquakes (1255, $1408,1505,1833,1934$, and 2015) with magnitudes equal to or greater than 7.6 and several strong earthquakes since 1255 (Thapa et al. 2017) and have caused serious disasters with huge human casualties and countless monetary losses. The latest most destructive earthquake ( $\mathrm{Mw}=7.8$, 25 April 2015 Gorkha earthquake) and its accompanied aftershocks caused a high toll of casualties $(>8,600$ deaths and injuries $>20,000)$, damage ( $>5$ million houses) and monetary losses ( $~ 7$ billion in US dollars) in Nepal (Government of Nepal 2015). The mapping of active faults (e.g., Nakata 1972, 1982, 1989; Nakata et al. 1984; Dasgupta et al. 1987; Upreti et al. 2000; Nakata and Kumahara 2002; Taylor and Yin 2009; Styron et al. 2010) clearly shows earthquake potential in Nepal and the surrounding region. Until recently, an update of earthquakes catalogue in this region is received a very little attention. However, some studies (Thapa 2008; Thapa and Wang 2013; Thapa et al. 2017) have well-documented past seismic activity of Nepal and the surrounding region in the course of preparing the seismic ground motion hazard maps of Nepal. This study focuses on updating and analyzing an earthquakes catalogue for Nepal and the surrounding region spanning the period from 1255 to 2015 . The present study assembles and updates the catalogue of earthquakes occurring in Nepal and surrounding region $\left(26^{\circ}-31.7^{\circ} \mathrm{N}, 79^{\circ}-90^{\circ} \mathrm{E}\right)$ spanning the period from 1255 to 2015 . It analyzes and presents a complete picture

Corresponding author

Email: dilliramthapa14@hotmail.com (D.R. Thapa) of the spatial and temporal distribution patterns of Nepalese earthquakes occurring between 1255 and 2015. The analysis of spatio-temporal patterns of seismicity of Nepal and the surrounding region is essential for hazard assessment of the country.

\section{DATA ANALYSIS}

For this study, seismicity data for considered region $\left(26^{\circ}\right.$ $31.7^{\circ} \mathrm{N}, 79^{\circ}-90^{\circ} \mathrm{E}$ ) have been assembled by combining earthquake data from previous studies (Thapa 2008; Thapa and Wang 2010; Thapa and Wang 2013) as well as data from the National Seismological Center (NSC), the International Seismological Centre (ISC), and the United States National Earthquake Information Centre (NEIC). These compiled seismicity datasets are carefully analyzed by removing repeated events and aftershocks and converted into a homogeneous surface wave magnitude scale (Ms) as in the previous earthquake catalogue analysis (Thapa and Wang 2013; Thapa 2014). This new homogeneous seismicity catalogue contains 981 earthquakes with surface wave magnitudes equal to or greater than 4.0. It comprises 788 earthquakes between magnitude 4.0 and 4.9, 135 earthquakes between magnitude 5.0 and 5.9, 43 earthquakes between magnitude 6.0 and 6.9, 13 earthquakes between magnitude 7.0 and 7.9 and two earthquakes between magnitude 8.0 and 8.9 .

\section{RESULTS OF SEISMICITY ANALYSIS}

The updated homogeneous earthquakes catalogue is separately analyzed both temporally and spatially herein in order to observe the patterns of seismicity in time and space. The observed spatial and temporal distributions patterns of seismicity in this study are presented in Figs. 1 and 2, respectively. 


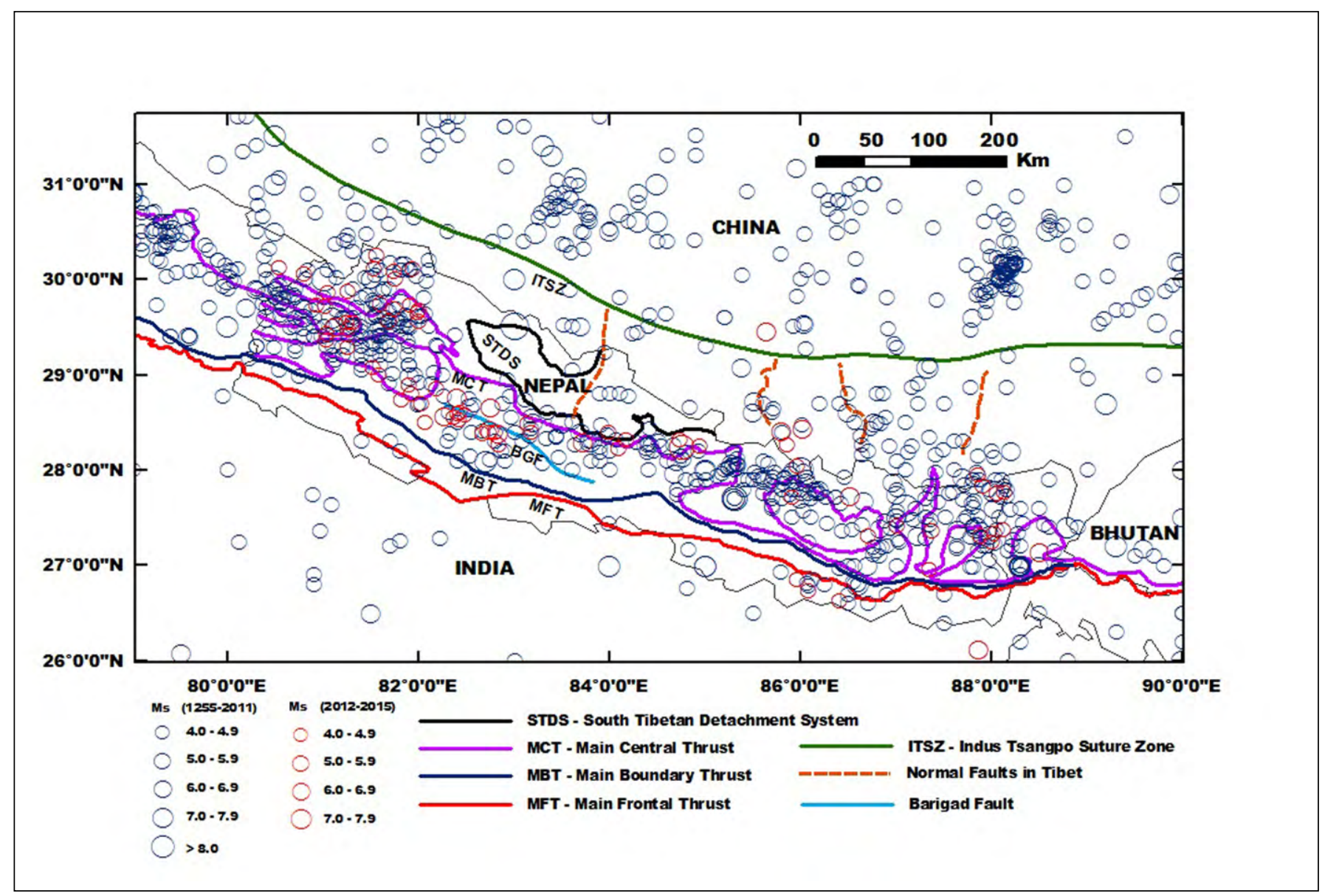

Fig. 1: Spatial distribution of earthquakes (Ms $\geq 4.0$ ) occurring in Nepal and the surrounding region between 1255 and 2015 . Major Faults (ITSZ: Indus-Tsangpo Suture Zone; STDS: South Tibetan Detachment System; MCT: Main Central Thrust; MBT: Main Boundary Thrust; and MFT: Main Frontal Thrust; and BGF: Bari Gad Fault). STDS, MCT, MBT, MFT and BGF in Nepal modified from Amatya and Jnawali (1994), ITSZ and active normal faults (Red discontinuous lines) in southern Tibet modified from Taylor and Yin (2009) and Thapa et al. (2018). Blue and red circles are the epicenters of earthquakes occurring between 1255 and 2011, 2012 and 2015 respectively.

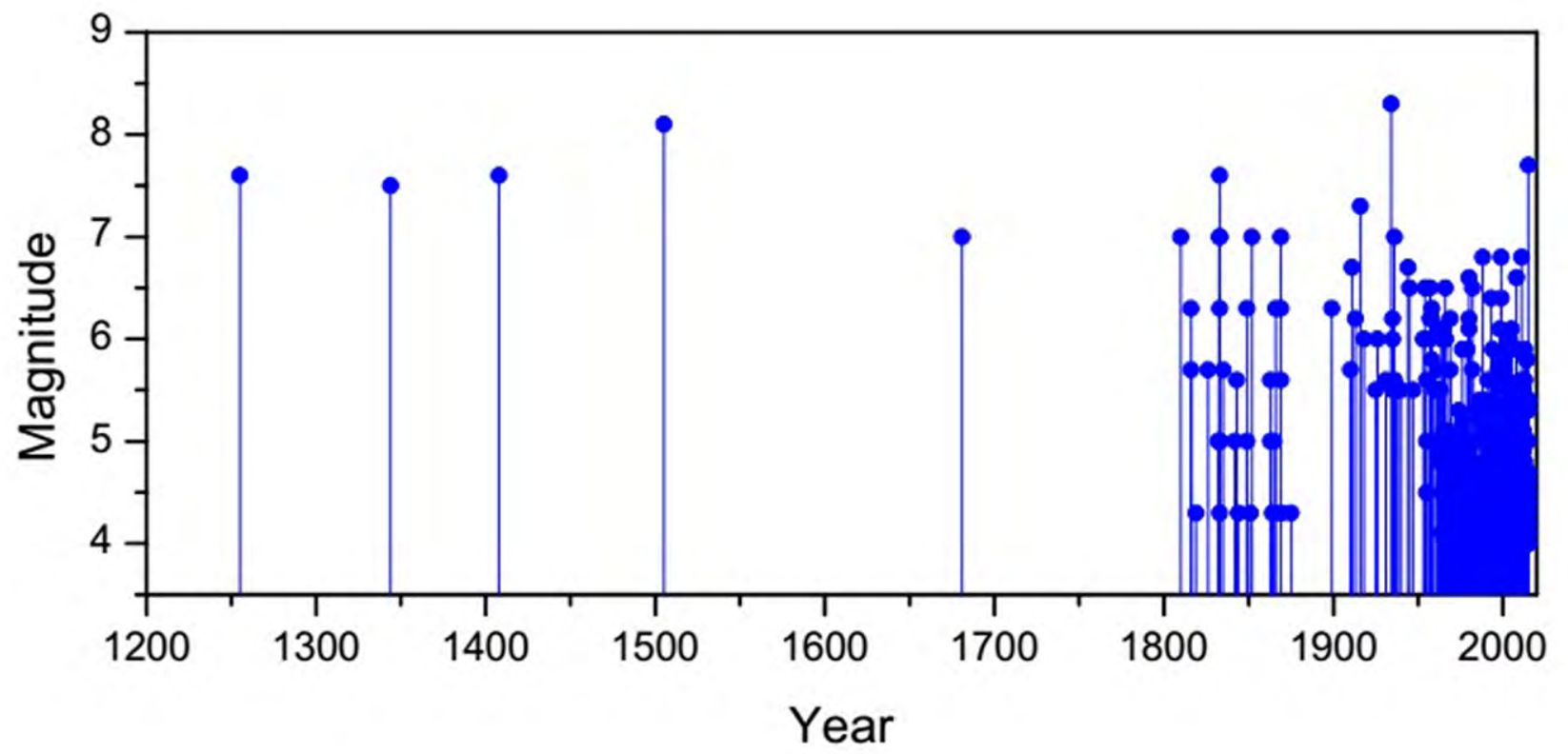

Fig. 2: Temporal distribution of earthquakes ( $\mathrm{Ms} \geq 4.0)$ occurring in Nepal and the surrounding region between 1255 and 2015. 


\section{Spatial distribution of earthquakes}

Fig. 1 shows the spatial distribution of epicenters of catalogued earthquakes covering the location between latitudes $26^{\circ} \mathrm{N}-31.7^{\circ} \mathrm{N}$ and longitudes $79^{\circ} \mathrm{E}-90^{\circ} \mathrm{E}$ for the period from 1255 to 2015 . One can see from the figure that earthquakes epicenters are not evenly distributed and demonstrate an approximately east-northwest trend in Nepal and the surrounding region. The uneven spatial distribution of earthquakes epicenters depicts distinctly higher earthquake activity in the eastern and far-western parts of Nepal than the southern portion of the country. It is pointed here that the epicenters of catalogued earthquakes is largely distributed along a continuous east-northwest trending about 50-150-kmwide belt throughout Nepal. The width of this seismic belt varies widely in the north-south portion with about $50-\mathrm{km}$ wide in the central segment and about $150-\mathrm{km}$-wide in the far western and eastern sections of the country.

In order to examine the spatial distribution of earthquakes epicenters and the surface traces of principle faulting structures in the considered region, the epicenter map of updated catalogued earthquakes is superimposed with principle known faults in Nepal and the surrounding area. Fig. 1 also displays the epicenter distribution of catalogued earthquakes spanning the period from 1255 to 2015 and principle known geological structures in Nepal and the surrounding area. The epicenter points in the figure also indicates that earthquakes are aligned parallel to the surface traces of the previously mapped principle faults (MFT, MBT, MCT, and STDS) in Nepal and the surrounding region. It can also be observed that the overwhelming majority of the recorded seismic events in Nepal and the surrounding region are aligned roughly in eastnorthwest trending direction and concentrated near the surface trace of Main Central Thrust (MCT). Moreover, epicenters of a great majority of strong events $(M s \geq 6.0)$ are spatially located at small distance from the previously mapped major faults in Nepal and the surrounding area.

\section{Temporal distribution of earthquakes}

The final updated earthquake dataset of the considered region from 1255 to 2015 (760 years) shows the significant heterogeneity in both quantity and quality. Due to the heterogeneous nature of seismicity datasets in quality and quantity, the final earthquake dataset is broadly subdivided into three different time windows as below:
(1) Earthquakes prior to 1800
(2) Earthquakes during 1801-1963
(3) Earthquakes during 1964-2015

Fig. 2 demonstrates the temporal distribution of earthquakes with surface wave magnitudes equal to or greater than 4.0 occurred in Nepal and the surrounding region for the period between 1255 and 2015.

\section{Earthquakes prior to 1800}

There are very little accounts of documented historically damaging seismic events in Nepal within the time window prior to 1800 . The updated seismicity catalogue contains five historically documented catastrophic earthquakes $(M=7.6$, $1255 ; \mathrm{M}=7.5,1344 ; \mathrm{M}=7.6,1408 ; \mathrm{M}=8.1,1505$; and $\mathrm{M}=7.0$, 1681) with magnitudes equal to or greater than 7.0 in this time window. This time window does not consists of medium to strong magnitude earthquakes and cannot be considered for earthquake activity analysis, however catastrophic seismic events reported in this analysis prior to 1800 are quite informative.

\section{Earthquakes during 1801-1963}

The available seismicity database in the time window 1801-1963 is much more informative compared with that of the earthquakes documented within the time period prior to 1800. There is a considerable increase in the total number of events and high quality of reported seismicity datasets over this time window 1801-1963 (163 years). Earthquake datasets covering time window from 1801 to 1963 contains 74 earthquakes $(\mathrm{Ms} \geq 4)$ included two damaging earthquakes occurred in 1833 and 1934 and the catalogue comprised of 10 , 35,22 , and 7 seismic events in the magnitude range of 4.0-4.9, 5.0-5.9, 6.0-6.9, and 7.0-7.9, respectively. It is noticed that the database from 1803 to 1963 is particularly complete for strong earthquakes only, whereas medium magnitude earthquakes reported within the same time window are not complete.

\section{Earthquakes during 1964-2015}

There has been a considerable increase both in the number of earthquake datasets and quality in the past 52 years time window 1964-2015 particularly from 1964 onwards when a large set of recorded earthquake datasets became available on a world-wide basis from the world-wide extensively distributed seismic instrumentation. The considerable increase in earthquakes implying that the assembled earthquake catalogue can be considered as complete from 1964 to 2015 and it contains in total 902 earthquakes with surface-wave magnitude equal to or greater than 4.0 (779 earthquakes between magnitude 4.0 and 4.9, 102 earthquakes between magnitude 5.0 and 5.9, 20 earthquakes between magnitude 6.0 and 6.9, and one earthquake between magnitude 7.0 and 7.9).

\section{CONCLUSIONS}

A catalogue of earthquakes (in terms of surface-wave magnitude, $\mathrm{Ms} \geq 4.0$ ) for Nepal and the surrounding region $\left(26^{\circ}-31.7^{\circ} \mathrm{N}, 79^{\circ}-90^{\circ} \mathrm{E}\right)$ for the period from 1255 to 2015 is updated and their spatio-temporal distribution patterns are analyzed. The spatial distribution of catalogued earthquakes covering the period 1255-2015 clearly demonstrates the uneven spatial distribution of earthquakes epicenters with distinctly higher earthquake activity in the eastern and farwestern parts of Nepal than the southern portion of the country. The superimposed epicenter map with previously mapped major faults in Nepal and the surrounding area indicates that the epicenters of a great majority of earthquakes are located 
near the surface trace of Main Central Thrust (MCT), whereas the epicenters of a few number of earthquakes lies at small distances from the surface traces of the Main Frontal Thrust (MFT) and Main Boundary Thrust (MBT) in the region.

The temporal distribution of available earthquake datasets spanning the period between 1255 and 2015 indicates that there are very little accounts of significant earthquakes prior to 1800 . The temporal trend of the updated seismic catalogue clearly demonstrate the gradual increase in earthquake data during the time window 1801-1963 and considerable increase in number and quality of seismic events predominantly from 1964 onwards. The considerably increase in both number and quality of seismicity data in the last five decades suggesting that the updated earthquake catalogue can be considered complete from 1964 to 2015. Furthermore, this newly updated homogeneous earthquake catalogue for Nepal and the surrounding region covering the period from 1255 to 2015 are very useful for future seismotectonic investigations, and better quantification of seismic hazard in this region.

\section{REFERENCES}

Amatya, K.M., and Jnawali, B.N., 1994. Geological map of Nepal, 1:1000000 scale, Department of Mines and Geology, Kathmandu, ICIMOD, CDG, UNEP.

Central Bureau of Statistics (CBS), 2011. National Population and Housing Census (NPHC), National Report Government of Nepal. (http://cbs.gov.np), 278 p.

Dasgupta, S., Mukhopadhyay, M.j, and Nandy, D.R., 1987. Active transverse features in the central portion of the Himalaya. Tectonophysics v. 136, pp. 255-264. http://dx.doi. org/10.1016/0040-1951 (87) 90028-X.

Government of Nepal, 2015. Nepal Earthquake 2015: Post Disaster Needs Assessment. Key Findings, v. A., National Planning Commission, Nepal, 123 p.

International Seismological Centre (ISC), Bulletin, Thatcham, UK, (http://www.isc.ac.uk/Bull), last access January 2017.

Nakata, T., 1972. Geomorphic history and crustal movements of foothills of the Himalaya. Science Report, Tohoku University, v. 22, pp. 39-177.

Nakata, T., 1982. A photogrammetric study on active faults in the Nepal Himalayas. Jour. Nepal Geol. Soc. v. 2, pp. 67-80.
Nakata, T., Iwata, S., Yamanaka, H., Yagi, H., and Maemoku, H., 1984. Tectonic landforms of several active faults in the western Nepal Himalayas. Jour. Nepal Geol. Soc., v. 4, pp. 177-200.

Nakata, T., 1989. Active faults of the Himalayas of India and Nepal. Geol. Soc. America, Sp. paper,. v. 232, pp. 243-264.

Nakata, T., and Kumahara, Y., 2002. Active faulting across the Himalaya and its significance in the collision tectonics. Active Fault Research, v. 22, pp. 7-16.

National Earthquake Information Center (NEIC), United States Geological Survey, U.S.A, (http://neic.usgs.gov), last access January 2017.

National Seismological Centre (NSC), Department of Mines and Geology, Nepal, (http://seismonepal.gov.np), last access January 2017.

Styron, R., Taylor, M., and Okoronkwo, K., 2010. Database of active structures from the Indo-Asian collision. Eos, v. 91(20), pp. 181-182. doi:10.1029/2010EO200001.

Taylor, M.H., and Yin, A., 2009. Active structures of the HimalayanTibetan orogen and their relationships to earthquake distribution, contemporary strain field, and Cenozoic volcanism. Geosphere v. 5(3), pp. 199-214. doi: 10.1130/GES00217.1.

Thapa, D.R, 2008. Seismic hazard and ground motion in Nepal. Diploma Dissertation, The Abdus Salam International Centre for Theoretical Physics, Trieste, Italy (Unpublished), $60 \mathrm{p}$.

Thapa, D.R., and Wang, G., 2010. Spatial distribution of seismicity and possible disaster in the Nepal Himalaya and its surrounding region. Proceedings of the $9^{\text {th }}$ international conference on civil and environmental engineering, China Architecture and Building Press, v. 2(53), pp. 117-120.

Thapa, D.R., and Wang, G., 2013. Probabilistic seismic hazard analysis in Nepal. Earthqu. Eng. and Eng. v. 12(4), pp. 577586. doi:10.1007/s11803-013-0191-z.

Thapa D.R., 2014. Seismic Zoning for Nepal. Ph.D. Dissertation Dalian University of Technology, P.R. China, 144 p.

Thapa, D.R., Tao, X., Wang, G., and Fan, F., 2017. Deterministic seismic hazard assessment for Nepal. Sixteenth World Conference on Earthquake Engineering, Santiago, Chile, paper 730, pp. 1-8.

Thapa, D.R., Tao, X., Fan, F., and Tao, Z., 2018. Aftershock analysis of the 2015 Gorkha-Dolakha (central Nepal) earthquake doublet. Heliyon Earth Sci. Nat. Haz. v. 4(7), pp. 1-12. doi: 10.1016/j.heliyon.2018. e00678.

Upreti, B,N., Nakata, T., Kumahara, Y., Yagi, H., Okumura, K., Rockwell, T.K., Virdi, N.S., and Maemoku, H., 2000. The latest active faulting in southeast Nepal. Proceedings of the Hokudan International symposium and School on active faulting, January 17-26, 2000, Japan, pp. 533- 536. 